\title{
Produção de pessegueiro e nectarina no planalto médio do Rio Grande do Sul em anos de inverno ameno
}

\author{
Peach and nectarine yields in the medium planes of Rio Grande \\ do Sul, Brazil in years of soft winter
}

Alexandre Augusto Nienow ${ }^{1}$ Luiz Gustavo Floss ${ }^{2}$

\section{RESUMO}

$O$ estudo do comportamento produtivo de pessegueiros e nectarineiras visou obter informações no sentido de auxiliar na determinação dos cultivares e seleções mais promissores para a região do Planalto Médio do Rio Grande do Sul. Foram estudados doze cultivares e nove seleções de pessegueiro; dois cultivares e uma seleção de nectarineira, com exigencias em frio menor que $7,2{ }^{\circ} \mathrm{C}$ variando entre $150 \mathrm{e}$ 400 horas. A coleção, constituída de três plantas por cultivar, conduzidas no espaçamento $6 \times 3 \mathrm{~m}$, no sistema de vaso aberto, sem irrigação, encontrava-se, em 1997, iniciando o sexto ciclo vegetativo. Observadas três safras agrícolas (1997/98, 1998/ 99 e 1999/00), os resultados obtidos demonstraram que a antecipação em maior ou menor grau da floração, dependendo do cultivar ou seleção, decorrente da elevação das temperaturas no final de junho e em julho, repercutiram na data de início da colheita, antecipando em até 20 dias. Diversos cultivares e seleções apresentaram rendimentos satisfatórios, acima de 15 t ha $a^{-1}$, porém, para a indicação dos materiais mais promissores, levando em conta o clima atípico, os estudos deverão ter prosseguimento, em que pese os cvs. Chimarrita, Marli e Eldorado já serem amplamente cultivados na região.

Palavras-chave: Prunus persica, pêssego, nectarina cultivares, colheita.

\section{ABSTRACT}

The objective of this research was to evaluate peach and nectarine varieties, selection, and yield in the Planalto Médio Region of the State of Rio Grande do Sul, Brazil. A total of twelve peach cultivars and nine selections; two nectarine cultivars and one selection, with required temperature lower than $7,2^{\circ} \mathrm{C}$, raging from 150 and 400 hours, were evaluated. These studies were carried out in the 1997/98, 1998/99 and 1999/00 growing seasons. The experimental fruit trees consisted of three plants from each cultivar, which were grown at $6 \times 3 \mathrm{~m}$ from each other, conducted according to an open vase shape, and without irrigation. In 1997, the plants were at the beginning of the sixth growth cycle. The results for three grown seasons (1997/98, 1998/99, 1999/00) showed that the anticipation of blooming also caused up an anticipation of 20 days in the harvest time. This effect was variety or selection dependent and it was a result of the higher temperatures in late June and during July. Several fruit tree cultivars and selections provided

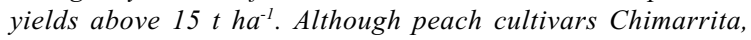
Marli and Eldorado are already widely grown in the Planalto Médio region, more studies are necessary to indicate the best materials, especially because of the weather conditions that ocurred in the last three seasons which were different from normal.

Key words: Prunus persica, cultivars, harvest.

\section{INTRODUÇÃO}

A cultura do pessegueiro, no Brasil, ocupa uma área superior a 23.000 hectares, com uma produção anual que ultrapassa 120 mil toneladas (MAIA et al., 1996). O principal Estado produtor é o Rio Grande do Sul, com mais de $60 \%$ da produção nacional (RAMALHO SOBRINHO \& GERALDO, 1997).

${ }^{1}$ Engenheiro Agrônomo, Doutor, Professor de Fruticultura da Faculdade de Agronomia e Medicina Veterinária (FAMV) da Universidade de Passo Fundo (UPF), CP 611, 99001-970, Passo Fundo, RS. E-mail: alexandre@upf.tche.br. Autor para correspondência.

${ }^{2}$ Engenheiro Agrônomo, ex-aluno da FAMV, UPF e ex-bolsista PIBIC-UPF 
A região do Planalto Médio, localizada no noroeste do Rio Grande do Sul, caracteriza-se pelo cultivo de grãos e a exploração da pecuária leiteira. Como alternativa de diversificação, a fruticultura tem se apresentado como excelente opção de investimento. São encontradas condições adequadas de clima e solo às culturas do pessegueiro e da nectarineira, exceto a ocorrência de geadas tardias, entre meados de agosto e início de setembro, que em alguns anos causam o abortamento de flores e frutos e a queima de órgãos em crescimento.

O conhecimento das condições climáticas são fundamentais na implantação de culturas de clima temperado, visto que interferem na formação das gemas floríferas e vegetativas, na quebra da dormência e no comportamento fenológico e produtivo.

A completa formação das gemas floríferas e vegetativas do pessegueiro e da nectarineira, e a quebra de dormência das plantas, exige um período de repouso das mesmas. Durante esse período, ocorrem transformações hormonais, convencionalmente medidas em número de horas de frio iguais ou inferiores a $7,2^{\circ} \mathrm{C}$, método clássico de medição (Weinberger, apud HERTER et al., 1998), sendo esta exigência de origem genética (Skinner, apud CAMELATTO, 1990). Além do acúmulo de frio, tem sido verificado que os genótipos apresentam uma necessidade de unidades de calor após a endodormência para iniciar o florescimento (RASEIRA \& MOORE, 1987; CITADIN, 1999).

Conforme SACHS \& HERTER (1984), geadas pouco antes, durante e depois do florescimento, constituem um dos sérios problemas, apresentandose o pistilo e as anteras como os órgãos mais sensíveis. $\mathrm{O}$ frio persistente pode causar distúrbios graves na polinização, no desenvolvimento do tubo polínico e na fusão dos núcleos femininos e masculinos, comprometendo a produção.

Este trabalho visou estudar os aspectos produtivos de 24 cultivares e seleções de pessegueiro e nectarineira produtores de frutos para o consumo in natura, contribuindo para a identificação dos materiais mais promissores para a região do Planalto Médio do RS. As condições do estudo foram de invernos mais amenos, que proporcionaram antecipação da floração, em parte por efeito dos fenômenos El Niño e La Niña.

\section{MATERIAL E MÉTODOS}

A coleção de pessegueiros e nectarineiras encontra-se instalada no Centro de Extensão e Pesquisa Agronômica (Cepagro), da Faculdade de Agronomia e Medicina Veterinária da Universidade de Passo
Fundo, RS, latitude $28^{\circ} 15^{\prime} 41^{\prime \prime} \mathrm{S}$, longitude $52^{\circ} 24^{\prime} 45^{\prime \prime} \mathrm{O}$, a $709 \mathrm{~m}$ de altitude.

O solo da área experimental pertence à unidade de mapeamento Passo Fundo, caracterizado como Latossolo Vermelho Distrófico típico. O número de horas de frio menor que $7^{\circ} \mathrm{C}$ ocorrido até agosto, nos anos de 1997, 1998 e 1999, foi de 198,9, 147,6 e 331,6 horas, respectivamente.

Foram estudados 12 cultivares de pessegueiro, alguns com conhecido requerimento de horas de frio (HF) menor ou igual a $7,2^{\circ} \mathrm{C}$ [Premier e Jóia (150 HF), Peach 16-33 e Sulina (200 HF), Chimarrita e Eldorado (250 HF), Marli (300 HF), Guaiaca (300-400 HF), Vila Nova (400 HF), Carmim, Purpúreo e Xavante] e 9 seleções (Cascata 415, Cascata 643, Cascata 647, Cascata 655 , Cascata 672 , Taquari 122 , Taquari 126 , Taquari 192 , Taquari 198); 2 cultivares de nectarineira (Linda e Sunlite) e 1 seleção (Necta 146). Os cvs. Sulina e Premier não foram estudados na safra 1997/98. Dentre estes, são mais conhecidos e cultivados no Rio Grande do Sul, em maior ou menor escala, os seguintes cultivares: Chimarrita, Eldorado, Guaiaca, Marli, Peach 16-33, Premier, Sulina e Vila Nova.

Para permitir a incorporação de novos materiais genéticos, os cultivares foram dispostos no campo seqüencialmente, representados por três ou cinco plantas. Os materiais estudados são oriundos da Embrapa/CPACT, Pelotas, RS, e da Estação Experimental da Fepagro, Veranópolis, RS. As copas foram enxertadas sobre o porta-enxerto cv. Capdeboscq, obtido de semente. O espaçamento de plantio é de $6 \times 3 \mathrm{~m}$ e as plantas foram conduzidas no sistema de vaso aberto, sem irrigação. Quando da primeira observação, em 1997, as plantas encontravam-se iniciando o sexto ciclo vegetativo.

A poda de frutificação consistiu na renovação dos ramos que produziram na safra anterior, podando após a primeira gema basal, e no desponte dos ramos que iriam produzir, com mais de $40 \mathrm{~cm}$ de comprimento, em $1 / 3$ do comprimento, com o objetivo de reduzir a carga de frutos. Os demais ramos não foram despontados.

O raleio dos frutos foi realizado quando atingiram 1,5 a 2,0 cm de diâmetro, antes do endurecimento do caroço, deixando cerca de $10 \mathrm{~cm}$ de distância entre os mesmos. Os tratamentos fitossanitários foram realizados de acordo com as necessidades, mediante observação e monitoramento permanente do pomar. As adubações de manutenção foram feitas em cobertura, sem incorporação, evitando o rompimento de raízes.

O comportamento produtivo foi determinado em três safras agrícolas (1997/98, 1998/99 e 1999/ 00), através da coleta dos seguintes dados: época de 
colheita, número de frutos por planta antes e após o raleio, peso médio dos frutos, produção por planta e por hectare.

O número de frutos após o raleio foi determinado por contagem na planta um mês antes da colheita. Uma amostra de 20 frutos por planta foi retirada para determinar o peso médio dos frutos. De posse deste dado e do número de frutos por planta, foi estimada a produção por planta.

Os dados coletados foram submetidos à análise da variância pelo teste $\mathrm{F}$, e as diferenças entre médias comparadas pelo teste de Scott-Knott, em nível de $5 \%$ de probabilidade de erro.

\section{RESULTADOS E DISCUSSÃO}

Nas safras estudadas, foram registradas condições meteorológicas atípicas para a região. Pode-se destacar, em 1997, a ocorrência de estiagem nos meses de março e abril. Verificou-se também, nas três safras estudadas, menor ocorrência de baixas temperaturas no final de junho e em julho, com flutuações de temperatura, provocando a antecipação do florescimento em até 15 dias, dependendo do grau de exigência em frio e calor para a saída da dormência de cada genótipo. Os anos de 1997 e 1998 caracterizaram-se pela baixa ocorrência de frio (198,9 e 147,6 horas de frio menor que 7,0 ${ }^{\circ} \mathrm{C}$ até agosto, respectivamente). Em 1999, embora tenha ocorrido até agosto uma quantidade total de horas de frio (331,6 horas) considerada quase normal para a região, que é de 365 horas (CUNHA, 1997), especificamente nos meses de junho e julho a ocorrência foi $44 \%$ inferior às normais, não evitando a floração precoce (NIENOW \& FLOSS, 2002). Estas condições decorreram, em parte, dos fenômenos El Niño (1997/98 e 1998/ 99) e La Niña (1999/00).

A floração mais precoce, principalmente, e a ocorrência de temperaturas médias mais elevadas durante o desenvolvimento dos frutos, anteciparam, em maior ou menor grau, as datas normais de início de colheita (Tabela 1). Como exemplos citam-se os pessegueiros 'Chimarrita' e 'Marli', que, em trabalho realizado por NIENOW \& LICODIEDOFF (1996), em Passo Fundo, apresentaram o início da colheita entre 02 e 07 de dezembro e 08 a 21 de dezembro, respectivamente, considerando as safras agrícolas de 1990/91, 1991/92 e 1992/93, ou seja, no mínimo 16 a 18 dias mais tarde.

De maneira geral, a seqüência dos cultivares e seleções mais e menos precoces foi mantido nas três safras. Destacaram-se como mais precoces os pessegueiros 'Peach 16-33', 'Sulina', 'Premier', 'Guaiaca' e 'Cascata 655', e as nectarineiras 'Linda' e 'Necta 146'. Os cultivares mais tardios foram os pessegueiros 'Vila Nova' e 'Eldorado'. A colheita estendeu-se por, aproximadamente, dois meses e meio, de meados de outubro à final de dezembro/início de janeiro (Tabela 1) .

Nas safras 1997/98 e 1998/99 não houve registro de geadas após o início da floração, possibilitando a produção de cultivares considerados precoces, normalmente não recomendados para a região. $\mathrm{Na}$ safra 1999/00, a ocorrência de uma forte geada em 13 de agosto $\left(-4{ }^{\circ} \mathrm{C}\right)$ comprometeu a produção da maioria dos genótipos estudados (Tabela 2), os quais encontravam-se em floração ou no início da frutificação. Ainda que a geada possa não ter sido considerada demasiadamente tardia para a região, o dano foi abrangente, em decorrência da antecipação da florada, anteriormente comentada.

Destacaram-se por proporcionar maior número de frutos após o raleio, na safra 1997/98 (Tabela 
Tabela 2 - Número de frutos por planta, antes e após o raleio, de cultivares e seleções de pessegueiros e nectarineiras - safras agrícolas 1997/98, 1998/99 e 1999/00, Passo Fundo, RS.

\begin{tabular}{|c|c|c|c|c|c|c|}
\hline \multirow{3}{*}{$\begin{array}{l}\text { Cultivares e } \\
\text { seleções }\end{array}$} & \multicolumn{6}{|c|}{ Número de frutos por planta } \\
\hline & \multicolumn{3}{|c|}{ Antes do raleio } & \multicolumn{3}{|c|}{ Após o raleio } \\
\hline & $1997 / 98$ & $1998 / 99$ & $1999 / 00$ & $1997 / 98$ & $1998 / 99$ & $1999 / 00$ \\
\hline Chimarrita & $1386 \mathrm{a}^{1}$ & $423 \mathrm{c}$ & $0 \mathrm{~d}$ & $545 \mathrm{a}$ & $177 \mathrm{~b}$ & $0 \mathrm{i}$ \\
\hline Cascata 655 & 1414 a & $701 \mathrm{~b}$ & $54 \mathrm{~d}$ & $503 \mathrm{a}$ & $332 \mathrm{a}$ & $54 \mathrm{~h}$ \\
\hline Vila Nova & $813 \mathrm{c}$ & $540 \mathrm{c}$ & $37 \mathrm{~d}$ & $494 \mathrm{a}$ & $291 \mathrm{a}$ & $37 \mathrm{~h}$ \\
\hline Taquari 122 & $1377 \mathrm{a}$ & $740 \mathrm{~b}$ & $354 \mathrm{c}$ & $471 \mathrm{a}$ & 299 a & $176 \mathrm{e}$ \\
\hline Cascata 647 & $991 \mathrm{c}$ & $267 \mathrm{~d}$ & $0 \mathrm{~d}$ & $438 \mathrm{a}$ & $159 \mathrm{~b}$ & $0 \mathrm{i}$ \\
\hline Cascata 643 & $1049 \mathrm{~b}$ & $271 \mathrm{~d}$ & $358 \mathrm{c}$ & $427 \mathrm{a}$ & $163 \mathrm{~b}$ & $107 \mathrm{~g}$ \\
\hline Taquari 192 & $895 \mathrm{c}$ & $495 \mathrm{c}$ & $0 \mathrm{~d}$ & $418 \mathrm{a}$ & $260 \mathrm{a}$ & $0 \mathrm{i}$ \\
\hline Purpúreo & $1044 \mathrm{~b}$ & $147 \mathrm{e}$ & $1837 \mathrm{a}$ & $360 \mathrm{~b}$ & $108 \mathrm{~b}$ & 739 a \\
\hline Cascata 672 & $1064 \mathrm{~b}$ & $0 \mathrm{f}$ & $161 \mathrm{~d}$ & $359 \mathrm{~b}$ & $0 \mathrm{~d}$ & $161 \mathrm{e}$ \\
\hline Sunlite $^{2}$ & $1136 \mathrm{~b}$ & $137 \mathrm{e}$ & $126 \mathrm{~d}$ & $358 \mathrm{~b}$ & $76 \mathrm{c}$ & $90 \mathrm{~g}$ \\
\hline Taquari 126 & $789 \mathrm{c}$ & $776 \mathrm{~b}$ & $0 \mathrm{~d}$ & $345 \mathrm{~b}$ & $363 a$ & $0 \mathrm{i}$ \\
\hline Eldorado & $777 \mathrm{c}$ & $553 \mathrm{c}$ & $241 \mathrm{~d}$ & $343 \mathrm{~b}$ & $299 \mathrm{a}$ & $173 \mathrm{e}$ \\
\hline Carmim & $936 \mathrm{c}$ & $120 \mathrm{e}$ & $1651 \mathrm{a}$ & $304 \mathrm{~b}$ & $72 \mathrm{c}$ & $559 \mathrm{~b}$ \\
\hline Guaiaca & $624 \mathrm{~d}$ & $585 \mathrm{~d}$ & $244 \mathrm{~d}$ & $297 \mathrm{~b}$ & $164 \mathrm{~b}$ & $193 \mathrm{e}$ \\
\hline Taquari 198 & $537 d$ & $520 \mathrm{c}$ & $48 \mathrm{~d}$ & $290 \mathrm{~b}$ & $290 \mathrm{a}$ & $48 \mathrm{~h}$ \\
\hline Marli & $676 \mathrm{c}$ & $190 \mathrm{e}$ & $837 \mathrm{~b}$ & $246 \mathrm{c}$ & $117 \mathrm{~b}$ & $262 \mathrm{~d}$ \\
\hline Linda $^{2}$ & $1513 \mathrm{~d}$ & $147 \mathrm{e}$ & $99 \mathrm{~d}$ & $229 \mathrm{c}$ & $107 \mathrm{~b}$ & $56 \mathrm{~h}$ \\
\hline Xavante & $555 \mathrm{~d}$ & $0 \mathrm{f}$ & $1755 \mathrm{a}$ & $196 \mathrm{c}$ & $0 \mathrm{~d}$ & $315 \mathrm{c}$ \\
\hline Jóia & $305 \mathrm{e}$ & $43 \mathrm{f}$ & $0 \mathrm{~d}$ & $173 \mathrm{c}$ & $43 d$ & $0 \mathrm{i}$ \\
\hline Premier & $221 \mathrm{e}$ & $705 \mathrm{~b}$ & $0 \mathrm{~d}$ & $140 \mathrm{c}$ & $355 \mathrm{a}$ & $0 \mathrm{i}$ \\
\hline Necta $146^{2}$ & $125 \mathrm{f}$ & $0 \mathrm{f}$ & $18 \mathrm{~d}$ & $102 \mathrm{~d}$ & $0 \mathrm{~d}$ & $18 \mathrm{i}$ \\
\hline Peach 16-33 & $117 \mathrm{f}$ & $722 \mathrm{~b}$ & $0 \mathrm{~d}$ & $71 \mathrm{~d}$ & $359 \mathrm{a}$ & $0 \mathrm{i}$ \\
\hline Sulina & $65 \mathrm{f}$ & $1258 \mathrm{a}$ & $0 \mathrm{~d}$ & $58 \mathrm{~d}$ & $367 \mathrm{a}$ & $0 \mathrm{i}$ \\
\hline Cascata 415 & $0 \mathrm{~g}$ & $21 \mathrm{f}$ & $388 \mathrm{c}$ & $0 \mathrm{e}$ & $21 \mathrm{~d}$ & $119 \mathrm{f}$ \\
\hline CV (\%) & 9,40 & 14,73 & 35,79 & 11,06 & 12,56 & 20,72 \\
\hline
\end{tabular}

${ }^{1}$ Médias não seguidas de mesma letra minúscula na coluna diferem entre si pelo teste de Scott Knott em nível de 5\% de probabilidade do erro.

${ }^{2}$ Nectarineiras.

2), os pessegueiros cvs. Chimarrita e Vila Nova, e as seleções Cascata 643, Cascata 647, Cascata 655, Taquari 122 e Taquari 192. Na safra 1998/99, destacaram-se os pessegueiros cvs. Sulina, Peach 16-33, Premier, Eldorado e Vila Nova, e as seleções Cascata 655, Taquari 122, Taquari 126, Taquari 192 e Taquari 198.

Constata-se, nos dados apresentados na Tabela 2, que o número de frutos na safra 1998/99 foi, de maneira geral, sensivelmente inferior ao obtido na safra anterior, devido ao aumento observado no abortamento de flores e frutos. $\mathrm{O}$ fato pode ser atribuído a alguns fatores como: menor número de horas de frio, que pode ter propiciado um maior número de gemas floríferas não perfeitamente formadas, abortando; deficiente brotação na fase de florescimento e início da frutificação, reduzindo a produção de fotoassimilados necessários para o vingamento dos frutos; e alta pluviosidade no período de floração de vários cultivares, impedindo a perfeita polinização e fecundação. Nos meses de julho e agosto de 1998, ocorreu 191,0 e $257,4 \mathrm{~mm}$ de chuva, respectivamente, portanto acima das normais observadas por CUNHA (1997), que é de $153,4 \mathrm{~mm}$ e $165,7 \mathrm{~mm}$, respectivamente.

Na safra 1999/00, prejudicada pela ocorrência de geada tardia, proporcionaram maior número de fru- tos após o raleio, em ordem decrescente, os pessegueiros cvs. Purpúreo, Carmim, Xavante e Marli (Tabela 2).

Os pessegueiros cvs. Eldorado, Vila Nova e Chimarrita, e as seleções Taquari 198 e Taquari 192, apresentaram frutos de maior peso médio na safra 1997/98, entre 107,3 e 131,0g (Tabela 3). Na safra 1998/99, novamente o cv. Chimarrita produziu frutos de maior peso médio $(117,0 \mathrm{~g})$, seguido dos cultivares e seleções que haviam se destacado na safra anterior, com exceção do Vila Nova. Também destacaram-se os cvs. Purpúreo, Carmim, Cascata 415, Cascata 643 e Cascata 647 , com frutos pesando entre 85,5 e 93,5g. Na safra 1999, o destaque foi para o cv. Marli e as seleções Taquari 122, Taquari 192, Taquari, 198, Cascata 643 e Cascata 655, seguidos do Eldorado e Guaiaca.

O peso médio dos frutos foi considerado, de maneira geral, satisfatório, concordando com os obtidos por outros autores, como NAKASU et al. (1989), para o cv. Eldorado; por RASEIRA \& NAKASU (1989), na descrição da nectarina 'Linda'; SIMONETTO etal.(1995), para os cultivares Peach 16-33, Chimarrita, Marli, Premier e Sulina; e NIENOW \& LICODIEDOFF (1996), para os cultivares Marli eChimarrita. A maior produção por planta e produtividade (Tabela 3), na safra 1997/98, foi proporcionada pelos pessegueiros cvs. Vila Nova e Chimarrita $(63,4 \mathrm{e}$ $61,3 \mathrm{~kg} \mathrm{pl}^{-1}$, respectivamente), seguidos do Taquari 192, Eldorado e Cascata $655\left(44,1\right.$ a 48,8 $\left.\mathrm{kg} \mathrm{pl}^{-1}\right)$. Considerando que comercialmente a produtividade média esperada deve ser superior a $15 \mathrm{t} \mathrm{ha}^{-1}$, podem ser destacadas ainda as seleções Taquari 122, Taquari 126, Taquari 198, Cascata 643, Cascata 647, Cascata 672 e o cv. Purpúreo. Segundo RASEIRA \& NAKASU (1998), o cv. Chimarrita obtém alta produtividade em Pelotas, RS, entre 50 e $65 \mathrm{~kg} \mathrm{pl}^{-1}$. Para o mesmo cultivar, em outro trabalho, NIENOW \& LIECODIEDOFF (1996) haviam obtido, em Passo Fundo, RS, 29,3kg pl-1, e SIMONETTO et al. (1995), em Veranópolis, RS, média de $27,8 \mathrm{~kg} \mathrm{pl}^{-1}$.

Na safra 1998/99, com produtividades entre 13,0 e 15,5 t ha-1 , além do 'Eldorado', 'Cascata 655' e 'Taquari 192', que estavam entre os cultivares mais produtivos na safra anterior, destacaram-se as seleções Taquari 122, Taquari 126 e Taquari 198. 
Tabela 3 - Peso médio de frutos, produção por planta e por hectare de cultivares e seleções de pessegueiros e nectarineiras - safras agrícolas 1997/98, 1998/99 e 1999/00, Passo Fundo, RS.

\begin{tabular}{|c|c|c|c|c|c|c|c|c|c|}
\hline \multirow{2}{*}{$\begin{array}{l}\text { Cultivares e } \\
\text { seleções }\end{array}$} & \multicolumn{3}{|c|}{ Peso médio de frutos $(\mathrm{g})$} & \multicolumn{3}{|c|}{ Produção por planta $(\mathrm{kg})$} & \multicolumn{3}{|c|}{ Produção por hectare (t) } \\
\hline & $1997 / 98$ & $1998 / 99$ & $1999 / 00$ & $1997 / 98$ & $1998 / 99$ & $1999 / 00$ & $1997 / 98$ & $1998 / 99$ & $1999 / 00$ \\
\hline Chimarrita & $107,3 \mathrm{a}^{1}$ & $117,0 \mathrm{a}$ & $0,0 \mathrm{i}$ & $61,3 \mathrm{a}$ & $20,7 \mathrm{~b}$ & $0,0 \mathrm{~g}$ & $34,0 \mathrm{a}$ & $11,5 \mathrm{~b}$ & $0,0 \mathrm{~g}$ \\
\hline Vila Nova & $125,7 \mathrm{a}$ & $71,7 \mathrm{~d}$ & $76,0 \mathrm{e}$ & $63,4 \mathrm{a}$ & $20,9 \mathrm{~b}$ & $2,8 \mathrm{f}$ & $35,2 \mathrm{a}$ & $11,6 \mathrm{~b}$ & $1,6 \mathrm{f}$ \\
\hline Taquari 192 & $116,0 \mathrm{a}$ & $89,7 \mathrm{~b}$ & $0,0 \mathrm{i}$ & $48,8 \mathrm{~b}$ & $23,4 \mathrm{a}$ & $0,0 \mathrm{~g}$ & $27,1 \mathrm{~b}$ & $13,0 \mathrm{a}$ & $0,0 \mathrm{~g}$ \\
\hline Eldorado & $130,7 \mathrm{a}$ & $91,8 \mathrm{~b}$ & $93,7 \mathrm{~b}$ & $45,1 \mathrm{~b}$ & $27,8 \mathrm{a}$ & $16,2 \mathrm{~d}$ & $25,0 \mathrm{~b}$ & $15,5 \mathrm{a}$ & $9,0 \mathrm{~d}$ \\
\hline Cascata 655 & $91,3 \mathrm{~b}$ & $78,3 \mathrm{c}$ & $95,2 \mathrm{a}$ & $44,1 \mathrm{~b}$ & 26,6 a & $5,1 \mathrm{f}$ & $24,5 \mathrm{~b}$ & $14,8 \mathrm{a}$ & $2,8 \mathrm{f}$ \\
\hline Taquari 122 & $84,0 \mathrm{~b}$ & $81,5 \mathrm{c}$ & $98,1 \mathrm{a}$ & $39,9 \mathrm{c}$ & $24,4 \mathrm{a}$ & $17,2 \mathrm{~d}$ & $22,1 \mathrm{c}$ & $13,5 \mathrm{a}$ & $9,6 \mathrm{~d}$ \\
\hline Cascata 647 & $87,7 \mathrm{~b}$ & $93,5 \mathrm{~b}$ & $0,0 \mathrm{i}$ & $31,6 \mathrm{c}$ & $15,4 \mathrm{c}$ & $0,0 \mathrm{~g}$ & $17,6 \mathrm{c}$ & $8,6 \mathrm{c}$ & $0,0 \mathrm{~g}$ \\
\hline Taquari 126 & $89,0 \mathrm{~b}$ & $5,5 \mathrm{c}$ & $0,0 \mathrm{i}$ & $31,2 \mathrm{c}$ & $27,6 \mathrm{a}$ & $0,0 \mathrm{~g}$ & $17,4 \mathrm{c}$ & $15,3 \mathrm{a}$ & $0,0 \mathrm{~g}$ \\
\hline Taquari 198 & $131,0 \mathrm{a}$ & $89,0 \mathrm{~b}$ & $94,4 \mathrm{a}$ & $30,2 \mathrm{c}$ & $25,9 \mathrm{a}$ & $4,6 \mathrm{f}$ & $16,7 \mathrm{c}$ & $14,4 \mathrm{a}$ & $2,5 \mathrm{f}$ \\
\hline Cascata 672 & $83,3 \mathrm{~b}$ & $0,0 \mathrm{~g}$ & $80,1 \mathrm{~d}$ & $30,0 \mathrm{c}$ & $0,0 \mathrm{~d}$ & $12,9 \mathrm{e}$ & $16,7 \mathrm{c}$ & $0,0 \mathrm{~d}$ & $7,2 \mathrm{e}$ \\
\hline Cascata 643 & $97,0 \mathrm{~b}$ & $88,7 \mathrm{~b}$ & $95,8 \mathrm{a}$ & $29,2 \mathrm{c}$ & $14,6 \mathrm{c}$ & $10,2 \mathrm{e}$ & $16,3 \mathrm{c}$ & $8,1 \mathrm{c}$ & $5,7 \mathrm{e}$ \\
\hline Purpúreo & $78,7 \mathrm{~b}$ & $87,3 \mathrm{~b}$ & $78,5 \mathrm{~d}$ & $28,1 \mathrm{c}$ & $9,4 \mathrm{c}$ & $57,9 \mathrm{a}$ & $15,6 \mathrm{c}$ & $5,2 \mathrm{c}$ & $32,2 \mathrm{a}$ \\
\hline Carmim & $74,0 \mathrm{~b}$ & $91,2 \mathrm{~b}$ & $72,9 \mathrm{f}$ & $2,5 \mathrm{~d}$ & $6,6 \mathrm{~d}$ & $40,8 \mathrm{~b}$ & $12,5 \mathrm{~d}$ & $3,7 \mathrm{~d}$ & $22,6 \mathrm{~b}$ \\
\hline Sunlite $^{2}$ & $54,0 \mathrm{c}$ & $77,3 \mathrm{c}$ & $70,0 \mathrm{~g}$ & $19,4 \mathrm{~d}$ & $5,9 \mathrm{~d}$ & $6,3 \mathrm{f}$ & $10,8 \mathrm{~d}$ & $3,3 \mathrm{~d}$ & $3,5 \mathrm{f}$ \\
\hline Guaiaca & $62,7 \mathrm{c}$ & $62,0 \mathrm{e}$ & $91,6 \mathrm{~b}$ & $18,6 \mathrm{~d}$ & $10,7 \mathrm{c}$ & $17,7 \mathrm{~d}$ & $10,4 \mathrm{~d}$ & $5,9 \mathrm{c}$ & $9,8 \mathrm{~d}$ \\
\hline Xavante & $85,0 \mathrm{~b}$ & $0,0 \mathrm{~g}$ & $73,9 \mathrm{f}$ & $17,3 \mathrm{~d}$ & $0,0 \mathrm{~d}$ & $23,3 \mathrm{c}$ & $9,6 \mathrm{~d}$ & $0,0 \mathrm{~d}$ & $12,9 \mathrm{c}$ \\
\hline Marli & $95,7 \mathrm{~b}$ & $83,0 \mathrm{c}$ & $96,9 \mathrm{a}$ & $15,9 \mathrm{~d}$ & $9,7 \mathrm{c}$ & $25,4 \mathrm{c}$ & $8,8 \mathrm{~d}$ & $5,4 \mathrm{c}$ & $14,1 \mathrm{c}$ \\
\hline Linda $^{2}$ & $62,3 \mathrm{c}$ & $59,3 \mathrm{e}$ & $73,3 \mathrm{f}$ & $14,3 \mathrm{~d}$ & $6,4 \mathrm{~d}$ & $4,1 \mathrm{f}$ & $7,9 \mathrm{~d}$ & $3,5 \mathrm{~d}$ & $2,3 \mathrm{f}$ \\
\hline Premier & $98,7 \mathrm{~b}$ & $58,3 \mathrm{e}$ & $0,0 \mathrm{i}$ & $13,7 \mathrm{~d}$ & $21,2 \mathrm{c}$ & $0,0 \mathrm{~g}$ & $7,6 \mathrm{~d}$ & $8,8 \mathrm{c}$ & $0,0 \mathrm{~g}$ \\
\hline Jóia & $78,7 \mathrm{~b}$ & $78,2 \mathrm{c}$ & $0,0 \mathrm{i}$ & $13,6 \mathrm{~d}$ & $3,7 \mathrm{~d}$ & $0,0 \mathrm{~g}$ & $7,5 \mathrm{~d}$ & $2,0 \mathrm{~d}$ & $0,0 \mathrm{~g}$ \\
\hline Cascata 415 & $51,0 \mathrm{c}$ & $85,5 \mathrm{~b}$ & $85,7 \mathrm{c}$ & $9,7 \mathrm{~d}$ & $1,9 \mathrm{~d}$ & $10,2 \mathrm{e}$ & $5,4 \mathrm{~d}$ & $1,0 \mathrm{~d}$ & $6,7 \mathrm{e}$ \\
\hline Peach 16-33 & $97,7 \mathrm{~b}$ & $63,2 \mathrm{e}$ & $0,0 \mathrm{i}$ & $6,9 \mathrm{~d}$ & $24,9 \mathrm{~b}$ & $0,0 \mathrm{~g}$ & $3,9 \mathrm{~d}$ & $10,4 \mathrm{~b}$ & $0,0 \mathrm{~g}$ \\
\hline Necta $146^{2}$ & $79,7 \mathrm{~b}$ & $0,0 \mathrm{~g}$ & $66,2 \mathrm{~h}$ & $6,3 \mathrm{~d}$ & $0,0 \mathrm{~d}$ & $1,2 \mathrm{~g}$ & $3,5 \mathrm{~d}$ & $0,0 \mathrm{~d}$ & $0,7 \mathrm{~g}$ \\
\hline Sulina & $84,5 \mathrm{~b}$ & $42,0 \mathrm{f}$ & $0,0 \mathrm{i}$ & $4,9 \mathrm{~d}$ & $15,4 \mathrm{c}$ & $0,0 \mathrm{~g}$ & $2,7 \mathrm{~d}$ & $6,4 \mathrm{c}$ & $0,0 \mathrm{~g}$ \\
\hline CV (\%) & 17,0 & 5,5 & 2,8 & 40,7 & 23,9 & 20,7 & 40,7 & 23,9 & 20,7 \\
\hline
\end{tabular}

${ }^{1}$ Médias não seguidas de mesma letra minúscula na coluna diferem entre si pelo teste de Scott nott em nível de $5 \%$ de probabilidade do erro.

Na safra 1999/00, não sofreram danos pela geada ocorrida e proporcionaram maior produtividade o pessegueiro 'Purpúreo' $\left(57,9 \mathrm{~kg} \mathrm{pl}^{-1}\right)$, seguido do cv. Carmim $\left(40,8 \mathrm{~kg} \mathrm{pl}^{-1}\right)$ e, com produtividade inferior, os cvs. Marli e Xavante $\left(25,4\right.$ e 23,3 $\mathrm{kg} \mathrm{pl}^{-1}$, respectivamente). MEDEIROS \& RASEIRA (1998) afirmam que o cv. Marli, em condições favoráveis, produz mais de 40kg $\mathrm{pl}^{-1}$. SIMONETTO et al. (1995) obtiveram média de $45,3 \mathrm{~kg} \mathrm{pl}^{-1}$ e NIENOW \& LICODIEDOFF (1996) cerca de $46,9 \mathrm{~kg} \mathrm{pl}^{-1}$, superiores aos obtidos nas safras analisadas.

Entre as nectarineiras, as de melhor desempenho foram a 'Sunlite' e a 'Linda', especialmente a 'Sunlite', na safra 1997/98, entretanto, todas apresentaram frutos de tamanho considerado pequeno, abaixo de $80 \mathrm{~g}$.

Diversos cultivares e seleções apresentaram satisfatórios rendimentos, acima de $15 \mathrm{tha}^{-1}$, porém o clima atípico ocorrido, de menor acúmulo de frio e elevação das temperaturas em final de junho e em julho, tornam precipitada a indicação dos cultivares e seleções mais promissores, em que pese os cvs. Chimarrita, Marli e Eldorado serem já amplamente cultivados na região.

\section{CONCLUSÕES}

A antecipação em maior ou menor grau da floração dos pessegueiros e nectarineiras na região do
Planalto Médio do Rio Grande Sul, decorrente da elevação das temperaturas no final de junho e em julho, repercutiram na data de início da colheita, antecipando em até 20 dias, dependendo do cultivar ou seleção.

\section{REFERÊNCIAS BIBLIOGRÁFICAS}

CAMELATTO, D. Dormência em fruteiras de clima temperado. Horti Sul, Pelotas, v.1, n.3, p.12-17, 1990.

CITADIN, I. Necessidade de calor para antese e brotação em pessegueiro [Prunus persica (L.) Batsch]. 1999. 74f. Dissertação (Mestrado em Fruticultura de Clima Temperado) - Faculdade de Agronomia Eliseu Maciel, Universidade Federal de Pelotas.

CUNHA, G.R. da. Meteorologia: fatos \& mitos. Passo Fundo: Embrapa-CNPT, 1997. 268p.

HERTER, F.G.; SACHS, S.; FLORES, C.A. Condições edafoclimáticas para instalação do pomar. In: MEDEIROS, C.A.B.; RASEIRA, M. do C.B. (Eds.). A cultura do pessegueiro. Brasília : Embrapa-SPI; Pelotas:Embrapa-CPACT, 1998. p.20-28.

MAIA, A.L. et al. Produção e mercado de pera e pêssego no Brasil. Informações Econômicas, v.26, n.2, p.33-48. 1996.

NAKASU, B.H.; RASEIRA, M.C.B.; VENDRUSCULO, J.L.S. 'Eldorado', um pêssego com dupla finalidade. Horti Sul, Pelotas, v.1, n.0, p.18-20, 1989.

NIENOW, A.A., LICODIEDOFF, M.C. Comportamento fenológico e produtivo de cultivares de pessegueiro e nectarineira

Ciência Rural, v. 33, n. 2, mar-abr, 2003. 
no Planalto Médio do Rio Grande do Sul. Revista Brasileira de Fruticultura, Cruz das Almas, v.18, n.2, p.201-208, 1996.

NIENOW, A.A.; FLOSS, L.G. Floração de pessegueiros e necatrineiras no Planalto Médio do Rio Grande do Sul, influenciada pelas condições meteorológicas. Ciência Rural, Santa Maria, v.32, n.6, p.931-936, 2002.

RAMALHO SOBRINHO, R.; GERALDO, L.G. Aspectos econômicos da produção de pêssegos. Informe Agropecuário, Belo Horizonte, v. 18, n.189, p.5-7, 1997.

RASEIRA, M.C.B., MOORE, J.N. Time of flower bud initiation in peach cultivars differing in chilling requirement. Hortscience, Alexandria, v.22, n.2, p.216-218, 1987.
RASEIRA, M.C.B.; NAKASU, B.H. Cultivares: descrição e recomendação. In: MEDEIROS, C.A.B.; RASEIRA, M. do C.B. (Eds.). A cultura do pessegueiro. Brasília : Embrapa-SPI; Pelotas:Embrapa-CPACT, 1998. p.20-28.

SACHS, S.; HERTER, F.G. Localização do pomar. In: SACHS, S. et al. A cultura do pessegueiro. Pelotas : EmbrapaCNPFT, 1984. p.13-19. (Circular Técnica, 10).

SIMONETTO, P.R.; GRELLMANN, E.O.; SCHMIDT, E. Comportamento de cultivares de pêssego para mesa na região da Serra do nordeste do Rio Grande do Sul. Porto Alegre : Fepagro, 1995. 20p. (Circular Técnica, 8). 